\title{
Resistive switching in aluminum/anodized aluminum film structure without forming process
}

\author{
W. Zhu, ${ }^{1}$ T. P. Chen, ${ }^{1, a)}$ Z. Liu, ${ }^{1}$ M. Yang, ${ }^{1}$ Y. Liu, ${ }^{2}$ and S. Fung ${ }^{3}$ \\ ${ }^{1}$ School of Electrical and Electronic Engineering, Nanyang Technological University, Singapore 639798, \\ Singapore \\ ${ }^{2}$ State Key Laboratory of Electronic Thin Films and Integrated Devices, University of Electronic Science \\ and Technology of China, Chengdu, Sichuan 610054, People's Republic of China \\ ${ }^{3}$ Department of Physics, The University of Hong Kong, Hong Kong
}

(Received 1 September 2009; accepted 23 September 2009; published online 10 November 2009)

\begin{abstract}
Metal-insulator-metal (MIM) structure was fabricated by partially anodizing aluminum film followed by deposition of another aluminum film. Unipolar resistive switching between a high-resistance state and a low-resistance state with a high resistance ratio $\left(>\sim 10^{4}\right)$ was observed from the structure. The switching occurred without the requirement of a forming process, which was attributed to the pre-existing conductive filaments in the $\mathrm{Al}$-rich $\mathrm{Al}_{\mathrm{x}} \mathrm{O}_{\mathrm{y}}$ layer formed by the anodization. Each resistance state exhibited Ohmic behavior which could be explained by the metallic conduction and electron hopping from one isolated state to the next in the $\mathrm{Al}$-rich $\mathrm{Al}_{\mathrm{x}} \mathrm{O}_{\mathrm{y}}$ layer. The MIM structure showed good memory characteristics. (c) 2009 American Institute of Physics. [doi:10.1063/1.3253722]
\end{abstract}

\section{INTRODUCTION}

Resistive switching phenomenon in metal oxides such as $\mathrm{NiO},{ }^{1-4} \mathrm{TiO}_{2},{ }^{5,6} \mathrm{ZrO}_{2},{ }^{7} \mathrm{Cu}_{\mathrm{x}} \mathrm{O},{ }^{8}$ and $\mathrm{Al}_{\mathrm{x}} \mathrm{O}_{\mathrm{y}}{ }^{5,9}$ attracts great interest for a possible application in nonvolatile memory devices. Some complicated materials such as $\mathrm{Cr}$-doped $\mathrm{SrZrO}_{3}{ }^{10}$ and $\mathrm{V}$-doped $\mathrm{SrZrO}_{3}{ }^{11}$ also show the switching phenomenon, but their switching mechanisms could be different from each other. The resistance switching behavior could be explained by various mechanisms including charge trapping in dielectric layer, space-charge-limited conduction processes, ion conduction and electrodeposition, Mott transition, and conductive filaments (CFs). ${ }^{4}$ However, it is still difficult to use a complete physical model to describe switching phenomenon, as switching behavior may be defined by the dielectric materials, electrode materials, fabrication procedure, or even experiment setup. For most of the materials, a forming process, which is the first switching from initial state to low-resistance state (LRS), is usually required. The forming process in metal oxides could be a kind of soft breakdown in the oxide film. It is not desirable for practical applications as the "forming" voltage is usually much higher than the set voltage. In this work, unipolar resistive switching in a partially anodized aluminum film was observed. No forming process was required, which is attributed to the preexisting CFs formed by the excess aluminum in the Al-rich $\mathrm{Al}_{\mathrm{x}} \mathrm{O}_{\mathrm{y}}$ layer of the partially anodized aluminum film.

\section{EXPERIMENT}

A $10 \mathrm{~nm}$ thick silicon dioxide layer was first thermally grown on a p-type silicon substrate in order to protect the silicon substrate during the anodization. Then a $500 \mathrm{~nm}$ thick Al film was deposited on the silicon dioxide layer by dc sputtering of an $\mathrm{Al}$ target with purity of $99.99 \%$. Anodization

${ }^{a)}$ Electronic mail: echentp@ntu.edu.sg. of the $\mathrm{Al}$ film was undertaken in a $0.15 \mathrm{M} / \mathrm{L}$ solution of oxalic acid at $25^{\circ} \mathrm{C}$, by applying a constant voltage of $40 \mathrm{~V}$ using a Keithley 2400 source meter. A $2 \times 2 \mathrm{~cm}^{2}$ platinum mesh was used as the cathode. The anodizing time was about $120 \mathrm{~min}$. The chemical states of the film were analyzed by x-ray photoelectron spectroscopy (XPS) (Kratos AXIS Ultra) with monochromatic $\mathrm{Al} K \alpha(1486.71 \mathrm{eV})$ x-ray radiation. Figure 1 shows the XPS peak of Al $2 p$ core level taken from the film. The $\mathrm{Al} 2 p$ peak of the film can be decomposed into two components, i.e., metallic $\mathrm{Al}$ and $\mathrm{Al}_{2} \mathrm{O}_{3}$. The $\mathrm{Al}$ component indicates the existence of $\mathrm{Al}$-rich $\mathrm{Al}_{\mathrm{x}} \mathrm{O}_{\mathrm{y}}$ layer. A transmission electron microscope (TEM) image of the anodized aluminum film is shown in the inset of Fig. 1. The thickness of the $\mathrm{Al}_{\mathrm{x}} \mathrm{O}_{\mathrm{y}}$ film is about $30-50 \mathrm{~nm}$, as can be seen in the TEM image. A $200 \mathrm{~nm}$ thick aluminum film was then deposited on the anodized film as top electrode by electron beam

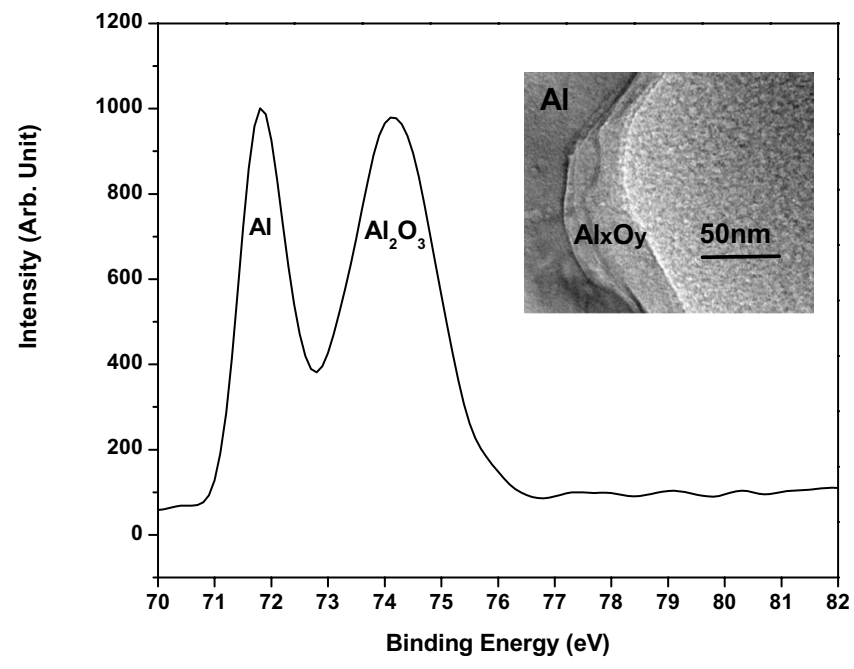

FIG. 1. XPS peak of Al $2 p$ core level taken from the anodized aluminum film. The inset shows the cross-sectional TEM image of the partially anodized Al film. 

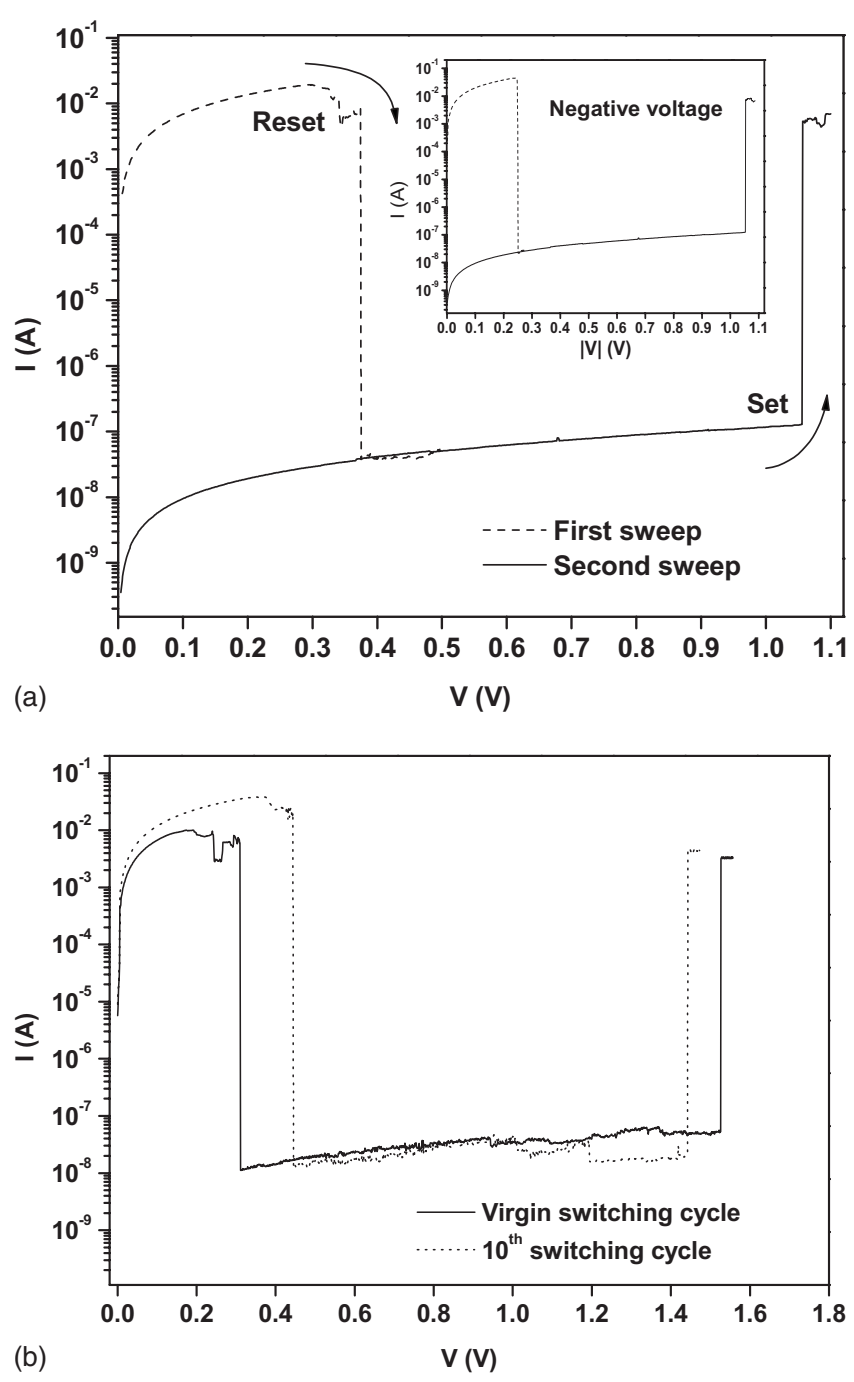

FIG. 2. (a) Resistive switching in the $\mathrm{Al} / \mathrm{Al}_{\mathrm{x}} \mathrm{O}_{\mathrm{y}} / \mathrm{Al}$ structure under positive bias. The inset shows the switching under negative bias. (b) Comparison of the virgin switching cycle with the tenth switching cycle.

evaporation to form the metal-insulator (i.e., the $\mathrm{Al}_{\mathrm{x}} \mathrm{O}_{\mathrm{y}}$ layer)-metal (i.e., the bottom unreacted Al layer) (MIM) structure. The diameter of the electrode is $200 \mu \mathrm{m}$. Currentvoltage $(I-V)$ measurements were carried out with a Keithley 4200 semiconductor characterization system at room temperature.

\section{RESULTS AND DISCUSSION}

Figure 2(a) shows the typical unipolar switching characteristics of the MIM structure. By sweeping the voltage applied to the top electrode from 0 to $+1.1 \mathrm{~V}$ with step of $1 \mathrm{mV}$, a bistable resistive switching was observed. As the biasing voltage was increased to a certain voltage $\left(V_{\text {reset }}\right)$ with a current compliance of $0.1 \mathrm{~A}$, a sudden drop of current appeared (the current dropped from $\sim 10^{-2}$ to $\sim 10^{-8} \mathrm{~A}$ ), i.e., the so-called reset process occurred, without any prior forming process. In the reset process the resistance was switched from a LRS to a high-resistance state (HRS), and then the sample maintained at the HRS. In the second voltage sweeping starting from $0 \mathrm{~V}$ with a smaller current compliance of $0.01 \mathrm{~A}$, the HRS was maintained until the voltage

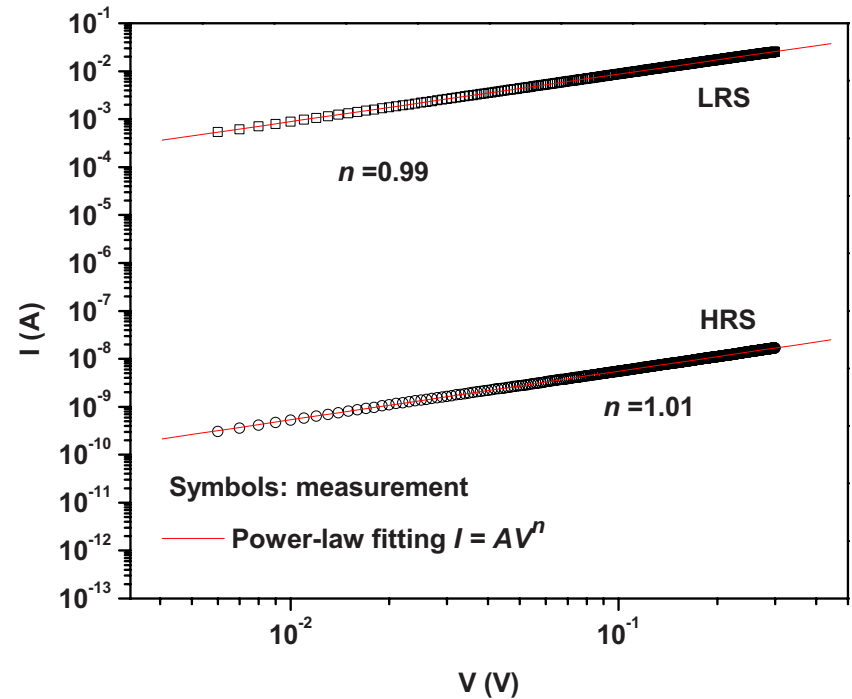

FIG. 3. (Color online) Plots of $\log I$ vs $\log V$ at low voltage region ( $V$ $<V_{\text {reset }}$ ) for the LRS and HRS.

was increased to certain value $\left(V_{\text {set }}\right)$ at which an abrupt increase in current appeared. This process is known as the "set" process and it is defined as the resistive transition from the HRS to the LRS. A smaller compliance current was used in the set process to avoid the hard breakdown in the oxide layer. The reset and set processes were highly reproducible. On the other hand, as shown in the inset of Fig. 2(a), similar reset and set processes were also observed in the negativevoltage sweeping. The independence of the reset and set processes on voltage polarity suggests that the interfaces of $\mathrm{Al}$ electrode $/ \mathrm{Al}_{\mathrm{x}} \mathrm{O}_{\mathrm{y}}$ and $\mathrm{Al}_{\mathrm{x}} \mathrm{O}_{\mathrm{y}} /$ unreacted $\mathrm{Al}$ did not play an important role and the switching was mainly determined by the $\mathrm{Al}_{\mathrm{x}} \mathrm{O}_{\mathrm{y}}$ layer. Figure 2(b) shows the comparison of the virgin switching cycle with the tenth switching cycle. As can be seen in the figure, the switching behavior is basically the same in the two cases. The result further confirms that the forming process is not necessary for switching to occur.

The switching behaviors observed in this work can be explained with the CF model. As revealed by the XPS analysis, there was a high concentration of metallic aluminum existing in the $\mathrm{Al}$-rich $\mathrm{Al}_{\mathrm{x}} \mathrm{O}_{\mathrm{y}}$ layer. The metallic aluminum preformed the CFs, yielding the initial LRS. This explains why no prior forming process was required for the reset/set processes. The pre-existing $\mathrm{CFs}$, which were formed by the excess aluminum in the $\mathrm{Al}_{\mathrm{x}} \mathrm{O}_{\mathrm{y}}$ layer, were alternatively connected and disconnected during the set/reset operation, and the situation could be somewhat different from that of other metal oxides. A high current was produced at a small voltage due to a large number of the pre-existing CFs. The sudden increase in resistance at the reset transition is attributed to the dissolution of some of the CFs as a consequence of Joule heating caused by the high current. In the set process, some of the broken CFs could be reconnected and or new CFs could be formed as a result of electrochemical reactions such as reduction reactions occurring in the $\mathrm{Al}$-rich $\mathrm{Al}_{\mathrm{x}} \mathrm{O}_{\mathrm{y}}$ layer under the influence of both the electric field and the electron injection from the cathode.

Figure 3 shows the $I-V$ characteristics (in log scale) of 


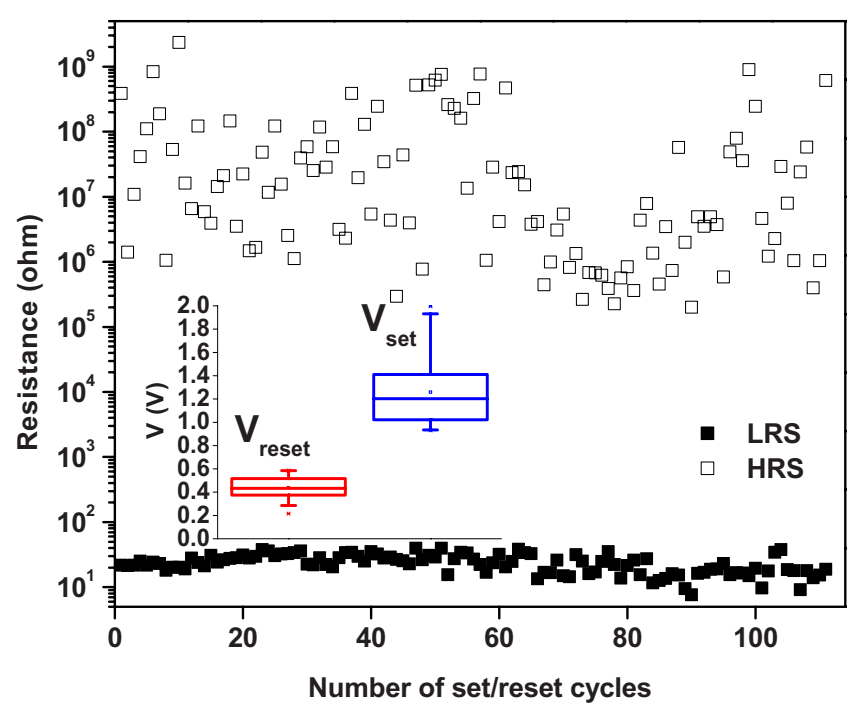

FIG. 4. (Color online) Variation in resistance for both the LRS and HRS with number of reset/set cycles. The resistance was measured at $0.1 \mathrm{~V}$. The inset shows the distributions of both $V_{\text {reset }}$ and $V_{\text {set }}$.

both the LRS and HRS for $V<V_{\text {reset }}$. In this low field region, both the LRS and HRS follow a power law, i.e., $I=A V^{n}$, where $n$ is an exponent factor $(n=1$ for perfect Ohmic conduction) and $A$ is a coefficient which depends on resistance state. As shown in Fig. 3, $n$ is very close to 1 for both the LRS and HRS, indicating that the CFs for both the LRS and HRS had an Ohmic behavior. A similar Ohmic conduction was reported in stoichiometric $\mathrm{ZrO}_{2}$ films. ${ }^{7}$ The Ohmic behavior could be explained by the following two mechanisms. Conduction in the form of nanoscale metal conductors was formed by a high concentration of excess metallic aluminum in the Al-rich $\mathrm{Al}_{\mathrm{x}} \mathrm{O}_{\mathrm{y}}$ layer. On the other hand, at low fields, current could be carried by thermally excited electrons hopping from one isolated state to the next in the Al-rich $\mathrm{Al}_{\mathrm{x}} \mathrm{O}_{\mathrm{y}}$ layer, which would exhibit an Ohmic behavior also. In other words, the two mechanisms were responsible for the current conduction. Both the LRS and HRS had the same conduction mechanisms, but they differed in the number and or the physical dimensions of the CFs. This argument is supported by the result shown in Fig. 3. As can be seen in the figure, both the LRS and HRS had about the same $n$ value, but they have a large difference in $A$ value (the $A$ value of the LRS is about six orders higher than that of the HRS). Therefore, the switching between the LRS and the HRS basically reflects the changes in the number and or the physical dimensions of the CFs.

Figure 4 shows the variation in resistance for both the LRS and HRS with the number of switching (set/reset) cycles. To minimize the influence of the electrical field during the resistance measurement, the resistance was measured at as low as $0.1 \mathrm{~V}\left(<V_{\text {reset }}\right)$. The resistance ratio of the HRS to the LRS is larger than $\sim 10^{4}$. The large memory window easily satisfies the requirement for distinguishing the two states in memory application. The inset of Fig. 4 shows the distributions of both $V_{\text {reset }}$ and $V_{\text {set }}$. The distribution of $V_{\text {reset }}$ was quite narrow (around $0.4-0.5 \mathrm{~V}$ ), the distribution of $V_{\text {set }}$ was relatively larger (around 1.0-1.4 V), but a window of $\sim 0.5 \mathrm{~V}$ between the $V_{\text {reset }}$ and the $V_{\text {set }}$ was still achieved. As

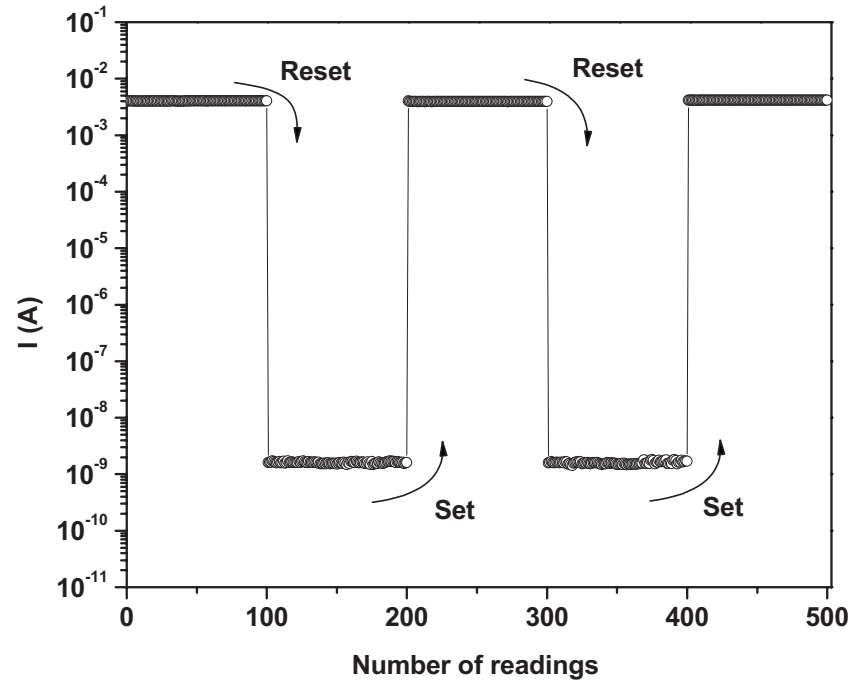

FIG. 5. Reading stability of both the LRS and HRS. The current was measured at $0.1 \mathrm{~V}$, and the time period corresponding to 100 readings is about $200 \mathrm{~s}$.

can be seen in Fig. 4, the resistance of the HRS was not as stable as that of the LRS, showing a larger fluctuation in the reset transition. However, both the HRS and the LRS had a good stability before switching occurring, as can be seen in Fig. 5 which shows the reading endurance for both the HRS and LRS. The results shown in Figs. 4 and 5 indicate that the MIM structure is promising in the resistive random access memory application.

\section{CONCLUSION}

In summary, the $\mathrm{Al}$-rich $\mathrm{Al}_{\mathrm{x}} \mathrm{O}_{\mathrm{y}}$ layer formed by partially anodizing $\mathrm{Al}$ thin film showed unipolar and repeatable switching between the LRS and the HRS with a high resistance ratio $\left(>\sim 10^{4}\right)$. The switching occurred without the requirement of a forming process, which is attributed to the pre-existing CFs in the Al-rich $\mathrm{Al}_{\mathrm{x}} \mathrm{O}_{\mathrm{y}}$ layer. Both the LRS and HRS in low voltage region exhibited Ohmic behavior which can be explained by the metallic conduction and electron hopping from one isolated state to the next in the Al-rich $\mathrm{Al}_{\mathrm{x}} \mathrm{O}_{\mathrm{y}}$ layer. The MIM structure consisting of the Al-rich $\mathrm{Al}_{\mathrm{x}} \mathrm{O}_{\mathrm{y}}$ layer showed good memory characteristics.

\section{ACKNOWLEDGMENTS}

This work has been financially supported by National Research Foundation of Singapore (NRF-G-CRP 2007-01). Y. Liu acknowledges National Natural Science Foundation of China (NSFC) under Project No. 60806040.

${ }^{1}$ D. Ielmini, C. Cagli, and F. Nardi, Appl. Phys. Lett. 94, 063511 (2009). ${ }^{2}$ D. C. Kim, S. Seo, S. E. Ahn, D.-S. Suh, M. J. Lee, B.-H. Park, and I. K. Yoo, Appl. Phys. Lett. 88, 202102 (2006).

${ }^{3}$ S. Seo, M. J. Lee, D. H. Seo, E. J. Jeoung, D.-S. Suh, Y. S. Joung, and I. K. Yoo, Appl. Phys. Lett. 85, 5655 (2004).

${ }^{4}$ U. Russo, D. Ielmini, C. Cagli, and A. L. Lacaita, IEEE Trans. Electron Devices 56, 186 (2009).

${ }^{5}$ K. M. Kim, B. J. Choi, B. W. Koo, S. Choi, D. S. Jeong, and C. S. Hwang, Electrochem. Solid-State Lett. 9, G343 (2006). 
${ }^{6}$ C. Rohde, B. J. Choi, D. S. Jeong, S. Choi, J.-S. Zhao, and C. S. Hwang, Appl. Phys. Lett. 86, 262907 (2005).

${ }^{7}$ X. Wu, P. Zhou, J. Li, L. Y. Chen, H. B. Lv, Y. Y. Lin, and T. A. Tang, Appl. Phys. Lett. 90, 183507 (2007).

${ }^{8}$ H. B. Lv, M. Yin, X. F. Fu, Y. L. Song, L. Tang, P. Zhou, C. H. Zhao, T. A. Tang, B. A. Chen, and Y. Y. Lin, IEEE Electron Device Lett. 29, 309
(2008).

${ }^{9}$ S. Kim and Y. K. Choi, Appl. Phys. Lett. 92, 223508 (2008).

${ }^{10}$ C. Y. Liu, P. H. Wu, A. Wang, W. Y. Jang, J. C. Young, K. Y. Chiu, and T. Y. Tseng, IEEE Electron Device Lett. 26, 351 (2005).

${ }^{11}$ C.-C. Lin, B.-C. Tu, C.-C. Lin, and C.-H. Lin, IEEE Electron Device Lett. 27, 725 (2006). 